\title{
Microscopic simulations of molecular cluster decay: Does the carrier gas affect evaporation?
}

\author{
Hoi Yu Tang and Ian J. Ford \\ Department of Physics and Astronomy, \\ University College London, Gower Street, \\ London WC1E 6BT, United Kingdom
}

\begin{abstract}
We develop a kinetic theory of cluster decay by considering the stochastic motion of molecules within an effective potential of mean force (PMF) due to the cluster. We perform molecular dynamics simulations on a 50-atom argon cluster to determine the mean radial force on a component atom and hence the confining potential of mean force. Comparisons between isolated clusters and clusters thermostatted through the presence of a 100-atom helium carrier gas show that the heat bath has only a slight effect upon the PMF. This confirms the validity of calculations of cluster properties using isolated cluster simulations. The PMF is used to calculate the atomic evaporation rate from these clusters, and results are compared with the predictions of the capillarity approximation together with detailed balance, both components of the classical theory of aerosol nucleation.
\end{abstract}




\section{INTRODUCTION}

Much is understood about the bulk states of matter known as solids, liquids and gases, but less is known regarding the phase transition of a system between these three states. ${ }^{1}$ All systems tend to arrange themselves so as to lower their energy, so when a new way of arranging the constituent atoms within the system allows it to lower this energy with respect to the old ordering, a phase transition occurs in which the atoms are rearranged on a grand scale. ${ }^{2}$ More precisely, thermodynamics dictates that isolated systems at externally fixed temperature and volume (or pressure) will adjust its internal properties so as to minimise its Helmholtz (or Gibbs) free energy, with such adjustments including the possibility of phase changes. ${ }^{3}$ In general, nucleation and decay events are responsible for the emergence of new phases within a system, be it through evaporation, condensation or crystallisation events. ${ }^{4,5}$

Due to the important role of nucleation in phase transitions, there exist a wide range of phenomena in nature and technology where knowledge of the underlying nucleation physics can help to understand and even model the systems in question. An example of a practical problem is the behaviour of steam in turbines, where water droplets produced through condensation in the transition from dry to wet steam can lead to the erosion of the turbine blades. ${ }^{6-8}$ Other examples include the development of decompression sickness in deep-sea divers, earthquakes, the formation of ice clusters in supercooled water, magma in volcanic eruptions, the foaming of plastics, bubbles in nuclear reactants, cryogenic systems, liquefied natural gas, and even in beer. ${ }^{9}$

The classical nucleation theory (CNT) was the earliest model of nucleation processes to emerge, having been developed from a combination of the most primitive ideas and known properties of the critical cluster over the past 70 years. ${ }^{10-12}$ Rather than attempting the laborious process of modelling all of the individual molecules and clusters within a system, CNT instead describes the evolution of the populations in each cluster size group. The usual starting point in the derivation of the classical theory is based upon transition state theory (TST), where the probability for some random fluctuation in a system at equilibrium is associated with a free energy fluctuation having a Maxwell-Boltzmann distribution. Classical theory identifies this probability with that of forming a condensation nucleus of $i$ molecules due to a fluctuation in the free energy of the system, and leads to the usual expression for the distribution of equilibrium cluster populations with respect to the monomer population $n_{1}^{e}$ :

$$
n_{i}^{e}=n_{1}^{e} \exp \left[-\Delta W_{i} / k T\right]
$$


where $W_{i}$ is the reversible cluster work of formation.

A crucial assumption made by classical nucleation theory is that no matter how small the critical clusters may be, they are ascribed the same equilibrium properties as that of a spherical droplet in the bulk condensed phase. This approximation is embodied in what is known as the capillarity approximation, usually expressed as

$$
W_{i}=A_{i} \sigma-i k T \ln S
$$

for a supersaturated vapour, where $A_{i}$ is the surface area of a spherical $i$-mer cluster, $\sigma$ is the surface tension, ${ }^{13} k$ is Boltzmann's constant, $T$ is the temperature, and $S$ is the supersaturation. The supersaturation itself is defined as $S=p_{v} / p_{v s}$, where $p_{v s}$ is the saturated vapour pressure at temperature $T$. The first term in this classical cluster work of formation represents an increase in Helmholtz free energy due to the formation of a spherical interface, and the second term a decrease of $k T \ln S$ per molecule due to the phase change from a supersaturated vapour to the condensed state. Starting from a supersaturated vapour, a phase change would require the growth of molecular clusters through stochastic collision and conglomeration events up to and beyond the critical cluster size $i^{*}$ at the maximum in the cluster work of formation. Classical theory works upon the basis that once a cluster has reached the critical size, it will then stand a good chance of continuing to grow into a macroscopic droplet of the condensed state.

The final and rather incomplete expression for the classical nucleation rate $J$ derived by Volmer and Weber $^{10}$ is simply

$$
J \propto \beta_{i^{*}} n_{1}^{e} \exp \left[-W_{i^{*}} / k T\right]
$$

where $\beta_{i}$ is the collision rate between a gas of monomers with a spherical $i$-cluster of surface area $A_{i}$, given by

$$
\beta_{i}=A_{i} \phi q=A_{i} \frac{p_{v}}{(2 \pi m k T)^{1 / 2}} q
$$

where $\phi$ is the molecular flux from kinetic theory, $p_{v}$ is the vapour pressure, $m$ is the molecular mass, and $q$ is the sticking factor of collisions (usually assumed to be unity). This expression for the classical nucleation rate is usually justified on the grounds that since critical clusters are those that have equal probabilities of either evaporating or growing into bulk droplets, it then stands to reason that the overall nucleation rate should be proportional the rate of attachment of monomers to clusters at the critical size $i^{*}$.

In 1935 Becker and Döring developed a kinetic interpretation on the evolution in cluster populations by taking into account their individual growth and decay rates. ${ }^{11}$ Given some distribution 
of cluster populations $\left\{n_{i}\right\}$ and applying the restriction that only those processes involving the loss or gain of one molecule $\left(\Delta n_{i}= \pm 1\right)$ be allowed, the evolution in the populations of any cluster size group can be expressed as

$$
\begin{aligned}
\frac{d n_{i}}{d t} & =\beta_{i-1} n_{i-1}+\gamma_{i+1} n_{i+1}-\beta_{i} n_{i}-\gamma_{i} n_{i} \\
& =J_{i-1}-J_{i}
\end{aligned}
$$

where $\beta_{i}$ and $\gamma_{i}$ are the respective growth and decay rate coefficients, and $J_{i}$ are the $i$-mer growth currents defined to be $J_{i}=\beta_{i} n_{i}-\gamma_{i+1} n_{i+1}$. Taking the steady state solution $\frac{d n_{i}}{d t}=0$ with the boundary conditions $\frac{d n_{1}}{d t}=0$ and $n_{M+1}=0$ to ensure a constant monomer population with some upper limit $M$ in the cluster size, the set of simultaneous equations

$$
J=\beta_{i} n_{i}-\gamma_{i+1} n_{i+1}
$$

emerge, which can then be solved to give the nucleation rate:

$$
J=\frac{\beta_{1} n_{1}}{1+\sum_{i=2}^{M} \prod_{j=2}^{i}\left(\gamma_{j} / \beta_{j}\right)}
$$

with the cluster growth rate coefficients $\beta_{i}$ taken to be the same as the cluster collision rates used in the original theory by Volmer and Weber (see Eq. (4)). Eq. (7) is usually referred to as the Becker-Döring solution to the set of kinetic equations of nucleation (Eq. (5)), and is a treatment which thus far remains completely general within the steady state situation.

The decay rate coefficients $\gamma_{i}$ can be determined by relating them to the known collision rates $\beta_{i}$, then taking the equilibrium case where $J=0$ so that Eq. (6) becomes the detailed balance relation:

$$
\gamma_{i+1} n_{i+1}^{e}=\beta_{i} n_{i}^{e}
$$

where the superscripts $e$ denote equilibrium properties of the system. Thus, the ratio of the growth to decay rate coefficients in the product sum of Eq. (7) may be expressed as the ratio of equilibrium populations between neighbouring cluster classes $\left(n_{i}^{e} / n_{i+1}^{e}\right)$, which can be simply calculated using statistical mechanics. ${ }^{11,12,14}$

Comparisons between this classical approach with experimental measurements on water from the past few decades have shown that CNT successfully predicts the dependence of nucleation rates upon the supersaturation ratio around $210-260 \mathrm{~K}$, but only to within a few orders of magnitude ${ }^{15-19}$ of the experimental values owing to an incorrect temperature-dependent behaviour. Although this 
may be considered a gross inaccuracy and possibly a serious failing in the theory, results from the classical theory are surprisingly accurate given the questionable application of the capillarity approximation underlying the classical theory. In fact, when the comparison is extended to a wider temperature range and to other substances, the discrepancy between the classical theory and experiment becomes even more apparent, ${ }^{20}$ showing that more than a small correction term to CNT is needed in order for it to correctly predict nucleation rates.

Attempts have been made to address several shortcomings within the CNT, such as the internally consistent classical theory of nucleation (ICCT),${ }^{21-23}$ the replacement free energy, ${ }^{24}$ and the volume (or length) scale correction by Reiss, Kegel and Katz. ${ }^{25}$ However, the ultimate usefulness of any corrections made upon the classical theory of nucleation are limited by the very nature of the classical theory and the questionable justification of the capillarity approximation. Furthermore, use of the classical theory to arrive at cluster decay rates based upon equilibrium statistical mechanics and detailed balance neglects the complex nature of cluster decay.

Alternatively, cluster decay rate coefficients $\gamma_{i}$ may be calculated via non-equilibrium molecular simulations using kinetic models ${ }^{26-28}$ in which individual evaporation events are counted over long simulations. Furthermore, computer simulations may also be used to determine general nucleation rates $J$ as well as the kinetic coefficients $\beta_{i}$ and $\gamma_{i}{ }^{29-33}$ Such kinetic modelling techniques have been made possible by the development of powerful computers, though their computational expense remains a limiting factor in the length and size of systems that can be conveniently modelled.

For reasons of simplicity and issues of computational resources, the majority of existing cluster calculations have been performed on isolated clusters. It is a temptation to be able to describe nucleation dynamics using only the properties of an isolated cluster, but would these results justifiably portray the properties of clusters in a carrier gas? How might the presence of a carrier gas be expected to affect the evaporation rate of a cluster? Certainly, a molecule escaping from the cluster could potentially collide with the carrier gas and be propelled back to rejoin the cluster. ${ }^{34}$ Conversely, collisions with the carrier gas may equally disturb the cluster to such a degree that evaporation events are encouraged, thus reducing the mean cluster decay rate. Therefore, interactions such as these between the cluster and carrier gas should ideally be accounted for in any attempt to calculate the properties of clusters in a heat bath. Toward this end, this study employs molecular dynamics calculations to simulate the evolution of a single argon cluster, both when isolated and when surrounded by a helium carrier gas. The advantages in employing molecular dynamics calculations lie in the dynamical modelling of all nucleation and decay events without having to explicitly identify each of them throughout the course of the simulations. 
In Sec. II we derive a kinetic theory of cluster decay in a general coordinate system with slight modifications upon the theory first developed by Ford and Harris ${ }^{28}$ followed in Sec. III by a brief discussion of the various definitions of the molecular cluster that have been developed. We investigate the effects of the carrier gas presence upon decay rates of an argon cluster in Sec. IV and Sec. VI using the kinetic theory of cluster decay, making comparisons against the classical theory prediction and results from another molecular simulations study. ${ }^{35}$ Most real cluster systems are surrounded by some carrier gas which acts as a heat bath and even a source of molecules for nucleation. Furthermore, in real systems there can exist large temperature and pressure gradients which should not sensibly be neglected and whose effects are not accounted for in the theory of homogeneous nucleation. Several groups have reported a drop in the nucleation rates with increasing carrier gas pressure ${ }^{36-45}$ though other experiments have shown no such dependence of the nucleation rate upon carrier gas pressures. ${ }^{46,47}$ The situation is further complicated by a theoretical study by Ford which suggested that there should be a non-linear dependence of the nucleation rate upon the carrier gas pressure. ${ }^{48}$ Another investigation attempted to place limits upon the magnitudes of the pressure effect using calculations based upon the classical theory of nucleation. ${ }^{49}$ However, experimental results have reported pressure dependencies outside of those limits. ${ }^{37-39}$ As such, the question of how and to what extent a carrier gas affects nucleation largely remains an unsolved problem.

\section{A KINETIC THEORY OF CLUSTER DECAY}

In the classical theory of nucleation, an explicit physical derivation of the cluster decay rate coefficient $\gamma_{i}$ is avoided by employing the detailed balance expression (Eq. (8)) and the collision rate $\beta_{i}$ (Eq. (4)). In the kinetic interpretation of cluster decay, individual molecules within a cluster are considered to reside in an effective potential well due to all the other cluster molecules and are driven by some stochastic force. ${ }^{50,51}$ Thus, when such a molecule manages to climb this potential well to reach such a distance that it is no longer under the influence of the cluster, it is then said to have escaped or evaporated. This kinetic approach to cluster decay was initially employed with a diffusion equation in energy space with results consistent with that from the classical nucleation theory for small supersaturations (large critical clusters). ${ }^{52,53}$ More recently however, a variation upon kinetic approach taken by Nowakowski et al was adopted in which the actual motion of cluster molecules in real space is considered, ${ }^{28}$ and it is in the spirit of the latter method that some of the results in this present work will be presented. 
The usual starting point in the kinetic model is a stochastic differential equation describing the one-dimensional motion of a molecule within a cluster:

$$
m \frac{\partial^{2} x}{\partial t^{2}}=f(x)-m \gamma \frac{\partial x}{\partial t}+\tilde{f}(t)
$$

where $x$ is the position, $f(x)$ is the mean force due to other molecules residing within the cluster, $\gamma$ is the friction coefficient, and $\tilde{f}(t)$ is a stochastic force with correlation function $\left\langle\tilde{f}\left(t^{\prime}\right) \tilde{f}(t)\right\rangle=(m B)^{2} \delta\left(t^{\prime}-t\right)$, a mean of $\langle\tilde{f}\rangle=0$ and variance $\left\langle\tilde{f}^{2}\right\rangle=\infty$. The constant $B$ here arises from consideration of the Langevin force $\Gamma(x, t)=\tilde{f}(t) / m$, whose correlation function is given by $\left\langle\Gamma\left(t^{\prime}\right) \Gamma(t)\right\rangle=B^{2} \delta\left(t^{\prime}-t\right)$ where $B^{2}=2 k T \gamma / m$.

The Langevin description shown above (Eq. (9)) can be simplified by taking the small mass (or high friction) limit, wherein the mass $m$ of the molecules in question are comparable to those of the gas or liquid within which they reside. Thus, the friction term can be considered to be far larger than the inertial term in the Langevin equation, and Eq. (9) becomes

$$
d x=-\frac{1}{\gamma m} \frac{d \Phi(x)}{d x} d t+\frac{1}{\gamma m} \int \tilde{f}(t) d t
$$

in integral form, where the mean force $f(x)$ has been replaced by the negative gradient in the potential of mean force $\Phi(x)$.

Using Itô calculus, the stochastic increment $\tilde{f}(t) d t$ can be replaced by an increment in some stochastic variable $d V$. It follows from the known properties of the stochastic force $\tilde{f}(t)$ that $d V$ must behave according to $\langle d V\rangle=0$ and $\left\langle(d V)^{2}\right\rangle=(m B)^{2} d t$. Furthermore, these properties of $d V$ also show that it may be interpretted as an increment in a Wiener process ${ }^{54}$ which in practice can be obtained via $d V=m B w(d t)^{1 / 2}$, where $w$ is a random number with a mean of $\langle w\rangle=0$, variance $\left\langle w^{2}\right\rangle=1$, and correlation $\left\langle w w^{\prime}\right\rangle=\delta_{w w^{\prime}}$.

Now, the stochastic differential equation can be written in a form which allows it to be converted into a Fokker-Planck equation that describes the time evolution of a probability distribution of the molecular positions. So, for each of the Cartesian components, this becomes

$$
\begin{aligned}
d \mathbf{s} & =\mathbf{a} d t+b\left[w^{\prime} \hat{\mathbf{i}}+w^{\prime \prime} \hat{\mathbf{j}}+w^{\prime \prime \prime} \hat{\mathbf{k}}\right](d t)^{1 / 2} \\
& =\mathbf{a} d t+b\left[d W^{\prime} \hat{\mathbf{i}}+d W^{\prime \prime} \hat{\mathbf{j}}+d W^{\prime \prime \prime} \hat{\mathbf{k}}\right]
\end{aligned}
$$

where $w(d t)^{1 / 2}$ has been replaced by an increment $d W$ in a dimensionless Wiener process, with

$$
\mathbf{a}=-\frac{1}{\gamma m} \nabla \Phi(x, y, z)
$$


and

$$
b=\frac{B}{\gamma}=\left(\frac{2 k T}{\gamma m}\right)^{1 / 2}
$$

The conversion into a Fokker-Planck form now requires the transformation of variables from $\mathbf{s}(x, y, z)$ into some function of $\mathbf{s}$ where $g(x, y, z)=g[\mathbf{s}(x, y, z)]$. Taking a Taylor expansion of $g$ and substituting for the increments $d x, d y$ and $d z$ from Eq. (11),

$$
\begin{aligned}
d g= & {\left[a_{x} \frac{\partial g}{\partial x}+\frac{b^{2}}{2} \frac{\partial^{2} g}{\partial x^{2}}\right] d t+b \frac{\partial g}{\partial x} d W^{\prime}+\left[a_{y} \frac{\partial g}{\partial y}+\frac{b^{2}}{2} \frac{\partial^{2} g}{\partial y^{2}}\right] d t+b \frac{\partial g}{\partial y} d W^{\prime \prime} } \\
& +\left[a_{z} \frac{\partial g}{\partial z}+\frac{b^{2}}{2} \frac{\partial^{2} g}{\partial z^{2}}\right] d t+b \frac{\partial g}{\partial z} d W^{\prime \prime \prime}
\end{aligned}
$$

since $\left\langle w^{2}\right\rangle=1$, and where cross terms from the Taylor expansion such as $d x d y, d y d z$ and $d z d x$ have vanished due to the independence of $w$ for each degree of freedom. Differentiating the mean $\langle g\rangle$ with respect to time, the stochastic $(d W)$ parts of Eq. (14) also vanish since $\langle d W\rangle=0$, giving

$$
\frac{d}{d t}\langle g\rangle=\left\langle a_{x} \frac{\partial g}{\partial x}+\frac{b^{2}}{2} \frac{\partial^{2} g}{\partial x^{2}}\right\rangle+\left\langle a_{y} \frac{\partial g}{\partial y}+\frac{b^{2}}{2} \frac{\partial^{2} g}{\partial y^{2}}\right\rangle+\left\langle a_{z} \frac{\partial g}{\partial z}+\frac{b^{2}}{2} \frac{\partial^{2} g}{\partial z^{2}}\right\rangle
$$

Alternatively, the left hand side of Eq. (15) can be expressed using the probability $P(x, y, z, t)$ of finding a molecule at position $(x, y, z)$ and time $t$, such that

$$
\begin{aligned}
\frac{d}{d t}\langle g\rangle & =\frac{d}{d t} \int_{-\infty}^{\infty} \int_{-\infty}^{\infty} \int_{-\infty}^{\infty} g P d x d y d z \\
& =\int_{-\infty}^{\infty} g \frac{\partial P}{\partial t} d^{3} \mathbf{r}
\end{aligned}
$$

where the integration limits covers all space.

Next, the substitution of Eq. (15) into the right hand side of Eq. (16) allows it to be separated into the simpler form

$$
\begin{aligned}
\frac{d}{d t}\langle g\rangle= & \int_{-\infty}^{\infty}\left(a_{x} P \frac{\partial g}{\partial x}+\frac{b^{2} P}{2} \frac{\partial^{2} g}{\partial x^{2}}\right) d^{3} \mathbf{r}+\int_{-\infty}^{\infty}\left(a_{y} P \frac{\partial g}{\partial y}+\frac{b^{2} P}{2} \frac{\partial^{2} g}{\partial y^{2}}\right) d^{3} \mathbf{r} \\
& +\int_{-\infty}^{\infty}\left(a_{z} P \frac{\partial g}{\partial z}+\frac{b^{2} P}{2} \frac{\partial^{2} g}{\partial z^{2}}\right) d^{3} \mathbf{r} \\
= & I_{x}+I_{y}+I_{z}
\end{aligned}
$$

where each term is dependent only upon one Cartesian component.

Taking the first term from the right hand side of Eq. (17), the integration with respect to $x$ can be performed by parts twice, whereupon the vanishing of surface terms since $P \rightarrow 0$ as $x \rightarrow \pm \infty$ leads to

$$
I_{x}=\int_{-\infty}^{\infty} g\left(-\frac{\partial\left(a_{x} P\right)}{\partial x}+\frac{1}{2} \frac{\partial^{2}\left(b^{2} P\right)}{\partial x^{2}}\right) d^{3} \mathbf{r}
$$


Similarly, the same procedure can be performed on $I_{y}$ and $I_{z}$, then allowing Eq. (17) to be rewritten in the form of Eq. (16), giving the general three dimensional Fokker-Planck equation

$$
\frac{\partial P}{\partial t}=-\nabla \cdot(\mathbf{a} P)+\frac{1}{2} \nabla^{2}\left(b^{2} P\right)
$$

Substitution of the prefactors a and $b$ (see Eq.s (12) and (13)) into Eq. (19) then gives the general Fokker-Planck equation describing the evolution of the probability distribution of molecules within a cluster:

$$
\frac{\partial P}{\partial t}=\frac{1}{\gamma m}\left[-\nabla \cdot(\mathbf{f} P)+k T \nabla^{2} P\right]
$$

where the mean force is now given by $\mathbf{f}=-\nabla \Phi(x, y, z)$ as opposed to the one dimensional form used earlier (see Eq. (10)).

Now, the model can be simplified into one more suitable for a molecular cluster by transforming the problem into spherical polar coordinates centred about the cluster centre of mass. Assuming that on average the molecular clusters are spherically symmetrical, then only the radial properties are needed, and the mean force becomes the mean radial force $f$, giving

$$
\frac{\partial P}{\partial t}=\frac{1}{\gamma m}\left[-\frac{1}{r^{2}} \frac{\partial}{\partial r}\left(r^{2} f P\right)+k T \frac{1}{r^{2}} \frac{\partial}{\partial r}\left(r^{2} \frac{\partial P}{\partial r}\right)\right]
$$

This Fokker-Planck equation differs from that used in a previous study by Ford and Harris, ${ }^{28}$ where the single-component Cartesian form of Eq. (20) was assumed at the beginning of the derivation in describing the radial dependence of cluster properties.

The rate of change in the probability distribution $P$ can be interpretted as the gradient in the probability current $\mathbf{J}(r)$ going away from the cluster centre of mass:

$$
\frac{\partial P}{\partial t}=-\nabla \cdot \mathbf{J}(r)=-\frac{1}{r^{2}} \frac{\partial}{\partial r}\left(r^{2} J\right)
$$

where as with the potential of mean force $\Phi(r)$ spherical symmetry in the probability distribution and current has been assumed.

Equating and rearranging Eqs. (21) and (22) gives the probability current as

$$
J=\frac{1}{\gamma m}\left(f P-k T \frac{\partial P}{\partial r}\right)
$$

after which taking the steady state solution where $J=0$ and integrating, gives

$$
-\int_{0}^{r} \frac{\partial \Phi}{\partial r} d r=k T \int_{0}^{r} \frac{1}{P} \frac{\partial P}{\partial r} d r
$$

which when evaluated leads to the relation:

$$
P(r) \propto \exp [-\Phi(r) / k T]
$$


However, for clusters exhibiting decay events, the probability current must necessarily be nonzero. It must also not be a constant quantity, since that would imply an infinite source of molecules at the cluster centre of mass.

Now taking the time-independent situation from Eq. (22) but with a variable current, the relation

$$
\frac{\partial P}{\partial t}=-\frac{1}{r^{2}} \frac{\partial}{\partial r}\left(r^{2} J\right)=0
$$

implies that $r^{2} J$ be constant. Although this also implies a relationship of $J(r) \propto r^{-2}$ which leads to the apparent paradox of having an infinite probability current at the cluster centre of mass, the time-independent solution is highly idealised as in reality there is a steady drain of probability, meaning $\partial P / \partial t<0$.

Next, the probability current can be rewritten in the form:

$$
J(r) \exp [\Phi(r) / k T]=-\frac{k T}{\gamma m} \frac{d}{d r}(P(r) \exp [\Phi(r) / k T])
$$

whereupon integration of both sides over $r$ from zero to some cutoff radius given by the boundary condition $P\left(r_{e}\right)=0$ leads to an expression for the probability current out of the boundary of a cluster at $r_{e}$ :

$$
J\left(r_{e}\right)=\frac{k T}{\gamma m} P(0)\left(r_{e}^{2} \int_{0}^{r_{e}} \frac{1}{r^{2}} \exp [(\Phi(r)-\Phi(0)) / k T] d r\right)^{-1}
$$

The probability distribution at the cluster centre of mass, $P(0)$, can be evaluated by normalising the steady state solution (see Eq. (25)) for all space where the probability is finite:

$$
P(r)=\exp [-\Phi(r) / k T]\left(\int_{0}^{r_{e}} \int_{0}^{2 \pi} \int_{0}^{\pi} \exp [-\Phi(r) / k T] r^{2} \sin \phi d r d \theta d \phi\right)^{-1}
$$

giving

$$
P(0)=\exp [-\Phi(0) / k T]\left(4 \pi \int_{0}^{r_{e}} r^{2} \exp [-\Phi(r) / k T] d r\right)^{-1}
$$

Finally, since $J\left(r_{e}\right)$ simply gives the probability current of an atom through a point at the edge of the cluster, the kinetically derived rate of cluster decay is completed by

$$
\gamma_{i}^{k i n}=4 \pi r_{e}^{2} i J\left(r_{e}\right)
$$

which, upon the additional insertion of an $\exp \left[-\Phi\left(r_{e}\right) / k T\right]$ term into both the numerator and denominator, gives the final kinetic decay rate as

$$
\gamma_{i}^{k i n}=\frac{i k T r_{e}^{2}}{\gamma m} \frac{\exp [-\Delta \Phi / k T]}{\left(\int_{0}^{r_{e}} r^{2} \exp [-(\Phi(r)-\Phi(0)) / k T] d r\right)\left(r_{e}^{2} \int_{0}^{r_{e}} \frac{1}{r^{2}} \exp \left[\left(\Phi(r)-\Phi\left(r_{e}\right)\right) / k T\right] d r\right)}
$$


The formulation of the kinetic theory of cluster decay presented here also differs from the treatment to the problem previously developed by Ford and Harris ${ }^{28}$ in that the time-independent probability current $J(r)$ is treated here as a function of the distance $r$ from the cluster of mass, rather than as a constant for all $r$. What results is a slight difference in the second integral in the final expression for the kinetic rate of cluster decay (see Eq. (32)) of $\left(r_{e} / r\right)^{2}$.

An important result here is the dependence of the kinetic decay rate upon the depth in the potential of mean force $\Delta \Phi$. The practical implications of this are that rather than having to perform laborious molecular dynamics simulations and individually counting the rare decay events, much shorter simulations can be used in order to adequately sample the potential of mean force $\Phi(r)$, as has been done in this study (see Sec. IV).

\section{DEFINITIONS OF MOLECULAR CLUSTERS}

Up to this point, the previous two sections have discussed and critiqued the developments of the classical nucleation theory and the kinetic theory of cluster decay. What is perhaps an equally fundamental issue that has not been mentioned thus far is the definition of a molecular cluster. At what point can a molecule be considered to have become part of, or escaped the cluster? Though not directly associated with the dynamics of nucleation and decay processes, the definition of what is a molecular cluster within each theory nevertheless bears heavily upon the outcome of calculations based upon models employing a microscopic description. In particular, we show in Sec. V that an adequate determination of the cluster centre of mass is crucial in preventing an undesirable smearing out of the potential of mean force, and can have direct consequences upon the calculation of cluster decay rates.

Most traditional methods of defining a cluster rely upon geometric constraints, such as a sphere of arbitrary radius centred around the centre of mass of a molecular cluster. ${ }^{55,56}$ Another common method of defining molecular clusters is to introduce some maximum allowable separation of molecules, within which neighbouring molecules are considered to be part of a cluster. This cutoff separation is usually defined with respect to the contact separation of the molecular species in its Lennard-Jones interaction parameter. Better known as the Stillinger criterion, ${ }^{57}$ this definition of molecular clusters has been employed in the current work, albeit with some minor modifications (see Sec. IV).

The two cluster definitions that have been described thus far are rather primitive and rely heavily upon human judgement in arriving at a suitable estimate for a cutoff for the cluster radius. One 
way in which progress has been made is in the form of a modified Stillinger cluster, ${ }^{58}$ where in addition to employing the Stillinger criterion, a spherical shell of volume $v$ is centred upon the centre of mass of the cluster of population $i$. In this definition, the cluster population $i$ and volume $v$ are considered to be equally important, and a lower limit in $i / v$ effectively places a lower limit upon which density fluctuations are to be considered full molecular clusters.

More recently, a dynamical approach to the cluster definition was developed by Harris and Ford $^{27}$ in which molecular dynamics simulations were used to follow the evolution of Lennard-Jones clusters. By studying a clear evaporation event back through simulation time to the point when the particle escapes the cluster, evaporation is identified at the moment in time when the total energy of the escaping molecule becomes positive in the centre of mass frame.

\section{MOLECULAR DYNAMICS SIMULATIONS}

An initial cluster configuration of 50 argon atoms is formed and equilibrated using molecular dynamics in the DL POLY code. ${ }^{59}$ Interatomic interactions are controlled using the Lennard-Jones potential $^{60}$

$$
U\left(r_{j k}\right)=4 \epsilon_{j k}\left[\left(\frac{\sigma_{j k}}{r_{j k}}\right)^{12}-\left(\frac{\sigma_{j k}}{r_{j k}}\right)^{6}\right]
$$

where $\epsilon_{j k}$ and $\sigma_{j k}$ are the potential well depth and contact separation parameters respectively ${ }^{61,62}$ (see Table I). A potential cut-off distance of $50 \AA$ is also implemented in the simulations.

Molecular dynamics simulations are performed with a 0.005 ps timestep using a the constant population, volume, and energy (NVE) microcanonical ensemble with a periodic cubic cell of edge length $100 \AA$ under the Verlet leapfrog scheme. The potential cut-off distance and cell dimensions are chosen so that the cell would be suitably large enough to prevent the argon cluster from interacting with mirror images of itself across the cell boundaries. Initially, the argon cluster is artificially heated by assigning the atoms velocities based upon the Maxwell-Boltzmann distributions for temperatures in the range $35 \mathrm{~K}$ to $50 \mathrm{~K}$ and equilibrated in molecular dynamics at the desired temperature. Upon equilibration, the clusters are left to evolve at their target temperatures for 50 ns in order to produce the data for later analysis.

Using the argon cluster as a template, a helium carrier gas is created in a cubic box of edge length $100 \AA$ centred around the cluster centre of mass. 100 helium atoms are placed at random within this box, with the proviso that no two helium atoms may be separated by a distance less than their contact separation defined by its Lennard-Jones potential parameter $\sigma_{H e H e}$. More importantly, 
in order to prevent the placement of a helium atom either too close to an argon atom or within the cluster itself, coordinates for the helium atoms are selected so that the separation between a helium and an argon atom is greater than its contact separation with argon $\sigma_{A r H e}$ (see Fig. 1). As with the isolated argon cluster, the helium atoms are assigned velocities according to the desired temperature, and the whole system is subsequently equilibrated prior to the 50 ns main calculation in the $100 \AA$ periodic cell.

In addition to calculations using a $100 \AA$ box, the same procedure with a carrier gas of 100 helium atoms is also applied to a smaller simulation cell of edge length $50 \AA$ with a reduced potential cutoff

of $17 \AA$. This makes possible simulations with carrier gas densities of $0.67 \mathrm{kgm}^{-3}$ and $5.70 \mathrm{kgm}^{-3}$ without the extra computational resources required for a larger number of carrier gas atoms. In the range of temperatures that the simulations covers, this corresponds to carrier gas partial pressures of $\sim 60 \mathrm{kPa}$ to $\sim 500 \mathrm{kPa}$ in the $100 \AA$ and $50 \AA$ simulation cells respectively. Again, care is taken in these choices of simulation cell sizes and their potential cutoff distances so that the cluster may not be able to interact with mirror images of itself across the periodic cell boundaries.

\section{ANALYSIS}

Due to the relatively small number of atoms within the systems, their mean simulation temperatures deviate slightly from the target temperature used in generating the initial velocities of the atoms. Therefore, the temperatures quoted in the remainder of this paper refer to the mean kinetic temperatures calculated after each molecular dynamics simulation. Given this, steady mean temperatures throughout the NVE simulations with fluctuations of the expected magnitudes confirm that the trajectories are ergodic and that the clusters are in thermal equilibrium (see Fig. 2).

System configurations and statistics are collected every 1000 simulation time steps, from which the mean radial force $f(r)$, potential of mean force (PMF) $\Phi(r)$, and the radial distribution functions can be calculated. Throughout the course of the simulations the argon clusters are seen to traverse their periodic cells as they evolve, meaning the centre of mass of the cluster never remains fixed with respect to the cell coordinates. Thus, in order to extract the most information for the radial force calculations from a molecular dynamics trajectory, the system in each configuration is shifted so that in each snapshot the cluster centre of mass lies at the origin of the cell. This ensures that the cluster potential of mean force is sampled evenly in all directions.

However, before these cell-centreing calculations can be performed, it is first necessary to define which argon atoms belong to the cluster, and those that have escaped from the cluster. Toward 
this end, the cluster definition used in this study is that of the Stillinger criterion described in Sec. III, with the condition that any argon atom having at least one other argon atom within a $1.5 \sigma_{A r A r}$ radius qualifies itself as a cluster atom. Obviously, as it stands this definition excludes the possibility of dimers, trimers and larger groupings away from the cluster. However, throughout the $50 \mathrm{~ns}$ simulations only single argon atoms are seen to escape and subsequently float freely around the system. When two free argon atoms do approach each other close enough so that their proximity qualifies them as being a Stillinger cluster, the two atoms remain unbound and thereafter continue along on their separate trajectories. Furthermore, since the cluster definition described above remains rather primitive, modifications on it are made based upon the dimensions of the 50atom argon cluster of interest here. The resulting additional constraints restrict atoms belonging to the cluster to within $15 \AA$ of the cluster centre of mass, with a maximum allowable argon-argon separation of $25 \AA$ to allow for a non-spherical cluster.

Such care is taken to accurately define the cluster centre of mass within the simulation cell due to the sensitivity in the radial force distribution upon correct placements of the cluster centre of mass. Given that the radial force $f(r)$ is calculated by

$$
f(r)=\mathbf{F}(x, y, z) \cdot \mathbf{r}(x, y, z)
$$

where $\mathbf{F}(x, y, z)$ is the force felt by an argon atom, and $\mathbf{r}(x, y, z)$ is the vector from the cluster centre of mass toward that atom, it is clear that the misplacement or imprecise calculation of the cluster centre of mass will give rise to incorrect calculations for the radial force. Moreover, in determining the radial force distribution by averaging over all configurations in the recorded trajectories, systematic errors in the radial force calculations will tend to undesirably smear and flatten out the radial force distribution.

In calculating the radial force distribution, configurations where only one argon atom has escaped are included in the calculations in order to sample the radial force beyond the cluster extent. Furthermore, configurations in which two or more argon atoms have escaped the cluster are ignored so that the form of the potential of mean force for a 50-atom argon cluster may be preserved.

Subsequently, the potentials of mean force $\Phi(r)$ for each cluster simulation are calculated by integrating the radial force distributions $f(r)$ :

$$
\Phi(r)=-\int_{0}^{r} f(r) d r=\int_{r}^{0} f(r) d r
$$

with the latter expression employed in order to satisfy the convention that $\Phi(r) \rightarrow 0$ as $r \rightarrow \infty$.

Even with the molecular dynamics simulations being $50 \mathrm{~ns}$ long, evaporation events are still rarely seen and result in a less than adequate sampling of the radial force at distances far from 
the cluster. Furthermore, not only do the infrequent argon-helium collisions at those radii give rise to a noisy radial distribution, but they ultimately appear as discontinuities in the corresponding potential of mean force. Given a sufficiently large sample of events at such distances, the influence of the cluster there is expected to be negligible and resemble results from isolated cluster calculations. Thus, analyses employing only the mutual argon interactions are adopted for the remainder of the calculations shown in this report. However, this raises the question of the validity in ignoring all argon-helium interactions since the effects of a helium carrier gas upon the argon cluster are exactly those that are of interest. This dilemma may be resolved by considering the fact that the effects of the heat bath upon the cluster will be reflected in the trajectories and positions of the argon atoms throughout the molecular dynamics simulations. Therefore, in this respect the determination of the potentials of mean force using only argon-argon interactions is not strictly equivalent to extracting the same properties given by isolated cluster calculations.

Finally, the radial distribution functions $n(r)$ and radial density profiles $\rho(r)$ centred about the cluster centres of mass in each simulation are calculated in order to detect any evidence of structure within the clusters, and also to ensure that the clusters had not frozen in the low temperatures of the molecular dynamics simulations. The radial positions of each argon atom from every configuration of the molecular dynamics simulation trajectories are tracked to find the cluster-centred radial distribution function, from which the radial density profiles are calculated using

$$
\rho(r) d r=\frac{n(r)}{4 \pi r^{2}}
$$

\section{RESULTS AND DISCUSSION}

Three sets of five molecular dynamics simulations have been performed for the isolated and thermostatted clusters at the carrier gas densities of $0.67 \mathrm{kgm}^{-3}$ and $5.70 \mathrm{kgm}^{-3}$ in the temperature region between $30 \mathrm{~K}$ and $53 \mathrm{~K}$. A clear temperature dependence is seen in the potentials of mean force $\Phi(r)$ (see Fig. 3), and as expected become shallower with increasing temperature, alluding to the destabilisation of the clusters at higher temperatures. In general, the washing out of the common features with higher temperatures seen in both the isolated and thermostatted clusters are an indication of some semblance of structure within the argon clusters, regardless of whether a carrier gas is present or not in the simulation. This is confirmed by the corresponding features in analyses of the cluster-centred radial distribution functions $n(r)$ and radial density profiles $\rho(r)$ of the isolated argon clusters (see Fig. 4), which suggest the existence of a shell-like structure to the liquid cluster - features that also become less prevalent with increasing temperature. Assuming 
that the cluster droplets have spherical geometries, then the number of atoms would be expected to grow as $\propto r^{2}$ with a constant density for at all radii. However, though this is not strictly the case owing to the shell-like structure evident in the cluster, a constant density droplet is approached with increasing temperature.

Another interesting property of the cluster is that of the cluster edge, or the point at which the potential of mean force can be considered to reach zero and where the cluster ceases to have any significant influence. Analyses show that the distance of the cluster edges do not change significantly across the different simulations (see Fig. 3), even though slight temperature dependencies are seen in the mean force distributions. Furthermore, this is all the more surprising given the clear expansion of the clusters due to heating (see Fig. 4), and suggests that stability of the clusters is not overtly affected by changes in outer regions of the cluster. An exception to the rule here can be seen in the isolated cluster simulation at $30 \mathrm{~K}$, whose potential of mean force reaches zero rather abruptly at a radius of $\sim 12 \AA$. Upon further inspection, it is found that at such low temperatures the cluster had exhibited no evaporation events over the $50 \mathrm{~ns}$ simulation. Consequently, the absence in any sampling of the radial force away from the cluster results in this lack of a smooth tail in the potential of mean force and the appearance of an unusually short cluster boundary.

In general, the results suggest that for a 50-atom argon cluster there exists some core region surrounded by two layers of atoms ending at the surface of the cluster. However, that is not to say that the cluster atoms are frozen to reside permanently within one of these regions, for the atoms are seen to freely explore a wide range of radii encompassing those "shells", as well as radii away from the cluster brought about by occasional evaporation events. If the minima in the potential of mean force represent favourable regions, or shells, for atoms to reside in, then they should correspond with features in the radial distribution functions, and such a correlation is indeed seen with the peaks at the radii of $\sim 3.5 \AA$ and $\sim 6.5 \AA$.

\section{A. Carrier gas effects}

In order to study the effects of the helium carrier gas upon the argon cluster, three sets of comparisons are made between the isolated and thermostatted clusters for similar temperatures in the regions of $37 \mathrm{~K}, 43 \mathrm{~K}$ and $48 \mathrm{~K}$. From here on these sets of comparisons shall respectively be referred to as the low, mid and high temperature data sets (see Figs. 5 to 7 ).

Initial studies of the radial force distributions from each cluster simulation suggest that the influence of the helium carrier gas is slight, with deviations within the margins of error associated 
with each calculation (see Fig. 5). However, further analyses of potentials of mean force (see Fig. 6) reveal more significant effects of the heat bath, particularly in the mid and high temperature molecular dynamics calculations. The presence of the helium carrier gas is negligible for the low temperature set of results, but in the two higher temperature sets of simulations the thermostatted clusters show a deeper potential of mean force, accompanied with radial distribution functions more representative of clusters at lower temperatures (see Fig. 7).

The combination of results thus far suggest that in both the mid and high temperature simulations the helium carrier gas produces a stabilising influence upon the argon clusters, with a larger effect seen in the middle temperature set of simulations. However, it is difficult to visually judge from the potentials of mean force alone the significance of this effect upon cluster stability, but used in conjunction with results from the kinetic theory of cluster decay, it is possible to make estimates of the cluster decay rate for each simulation.

\section{B. Cluster evaporation rates}

Using the results from the kinetic theory of cluster decay (see Eq. (32)), evaporation rates are calculated for each argon cluster based upon their potential of mean force profile (see Fig. 8). The value of the escape radius $r_{e}$ necessary to perform the numerical integration is estimated to be 17 $\AA$ for each cluster. In reality, this escape radius will most likely depend upon temperature, due to expansion of the cluster from heating as can be seen in these simulations and previous studies. ${ }^{28}$ However, in these calculations at least, the extent of this influence on the cluster is also seen to be largely independent within the temperature range which the molecular dynamics simulations are performed at. In addition to the decay rates and corresponding cluster lifetimes calculated from the molecular dynamics simulations, comparisons are made against the prediction made by classical nucleation theory and data from a separate molecular dynamics study by a group at the University of Cologne. ${ }^{35}$

In the molecular dynamics study by Kraska, ${ }^{35}$ a microcanonical NVE ensemble with a system similar to that in the current work was used, but with a larger system consisting up to thousands of argon atoms. Their investigations centred about the simulation of argon condensation at hugely accelerated rates through the use of large supersaturation ratios, and obtained estimates of critical cluster sizes $i^{*}$ and nucleation rates for various temperatures and supersaturations. It must be noted in interpreting their results that the temperature evolves throughout the nucleation period, thus possibly resulting in some uncertainty in the reported nucleating temperatures. Nevertheless, 
their reported critical cluster sizes can provide estimates of cluster decay rates using classical theory to compare with the single-cluster calculations presented here.

Since by definition the critical cluster is equally likely to either grow or decay, it follows that the cluster decay rate is simply the collision rate given by classical kinetic theory (see Eq. (4)), such that

$$
\gamma_{i^{*}}=\beta_{i^{*}}=\left(36 \pi i^{* 2} v_{l}^{2}\right)^{1 / 3} \frac{S p_{v s}}{(2 \pi m k T)^{1 / 2}} q
$$

where $v_{l}$ is the molecular volume ${ }^{63,64}$ and the temperature-dependent saturation vapour pressure from the Clausius-Clapeyron relation ${ }^{65}$ is given by

$$
p_{v s}=p_{0} \exp \left[\frac{Q}{k}\left(\frac{1}{T_{0}}-\frac{1}{T}\right)\right]
$$

with the reference pressure $p_{0}$ and temperature $T_{0}$ taken at the triple point of argon, ${ }^{66,67}$ and $Q$ the latent heat of evaporation. ${ }^{68}$

Starting from the detailed balance relation (see Eq. (8)), the classical cluster decay rate can be obtained via the capillarity approximation (see Eq. (2)) to give

$$
\gamma_{i}^{C N T}=\beta_{i-1} \exp \left[\sigma\left(36 \pi v_{l}^{2}\right)^{1 / 3}\left(i^{2 / 3}-(i-1)^{2 / 3}\right) / k T\right]
$$

where the same expression of the collision rate is used as that in the decay rate of the critical cluster (see Eq. (37)), but with $i=50$ in this instance.

Within the results obtained from this study alone, the decay rate and lifetime comparisons (see Fig. 8) show that there is little significant difference between the isolated argon clusters and those thermostatted by a helium carrier gas. However, initial analyses of the cluster potentials of the mean force (see Fig. 6) suggest that there may exist a non-negligible stabilisation of the cluster at the middle and high range of temperatures used in these simulations, with the lower of the two carrier gas densities providing a greater cluster stabilising effect. Cluster decay rate comparisons (see Fig. 8) would seem to corroborate this, with the $0.67 \mathrm{kgm}^{-3}$ carrier gas data (circles) lying below both the isolated cluster curve and that corresponding to the carrier gas at $5.70 \mathrm{kgm}^{-3}$.

Relative to the decay rates and obtained from the work by Kraska and from classical nucleation theory, the results of this study predominantly lie above both. Except for the coldest simulation of the isolated argon cluster at $30 \mathrm{~K}$, cluster decay rates calculated using the kinetic theory of cluster decay appear to agree well with the classical rates, lying within an order of magnitude above that of the classical prediction. Despite the good agreement however, it is rather strange that the classical theory predicts such similar cluster decay rates given its gross failure in describing the 
total nucleation rate. Although the failure of the classical theory may be attributed to mismatches of decay rates in the Becker-Döring ladder, the unexpected closeness of the results here suggest that this may not be the case for the 50-atom argon cluster. Another possible explanation may be absence of a factor representing the position of the cluster centre of mass ${ }^{25}$ mentioned in Sec. I. A recent paper by Kusaka ${ }^{69}$ on similar issues suggests an increase of the classical nucleation rate by a factor of between $10^{10}$ and $10^{12}$. However, even with this modification it still does not account for the huge discrepancies between classical theory and recent experimental results. ${ }^{20}$ Ultimately, it remains a puzzle as to why there is such a good agreement between the cluster decay rates calculated via the classical and kinetic theories, though similar studies such as that reported here performed upon a variety of cluster sizes should shed some light on the issue.

By contrast, the decay rates extracted from the Kraska study lie well below the other two sets of results, differing by about 3 and up to 6 orders of magnitude between the higher and lower range of temperatures respectively. The discrepancy between the Kraska decay rates and those calculated from the kinetic theory in this study as well as the classical prediction may be due to the artificially accelerated condensation simulations performed in their study, as under those high supersaturation conditions, it is likely that actual cluster decay rates during the simulations would have been extremely low.

\section{CONCLUSIONS}

We have explicitly developed a kinetic theory of cluster decay based upon the previous work by Ford and Harris ${ }^{28}$ but with several improvements regarding the Fokker-Planck treatment that lead to a slight modification in the expression for the cluster evaporation rate. More precisely, cluster decay rates calculated within the temperature range studied here are approximately $\sim 0.7$ of those calculated using the previous version of the theory. The results of the improved theory are ultimately used to determine the effects of a helium carrier gas upon the stability of an argon cluster in molecular dynamics simulations. Unlike other studies where large systems may be modelled, our approach simulates the evolution of a single cluster over a long period in order to determine cluster stabilities through their effective potential of mean force.

In comparison against isolated cluster calculations from a preceding study, ${ }^{28}$ our initial results had suggested a reduction the in depth of the cluster potential of mean force by up to half of that for the isolated cluster owing to carrier gas effects. At first sight this result may be surprising in that an inert carrier gas could destabilise the cluster to such an extent. Also, if such a large 
carrier gas effect indeed exists, it raises the question in the suitability of many previous studies that have used isolated cluster calculations to determine the properties of clusters in real thermostatted systems. However, upon closer inspection it is discovered that incorrect calculations of the radial force distribution are the cause, with inaccurate determination of the centre of mass of the clusters being the chief culprit. Hence, it is for this very reason that a significant amount of this current study has been devoted toward a suitable definition of argon clusters in the post-molecular dynamics analyses, for only then can the cluster centres of mass be adequately determined.

It is noted that if the argon clusters are close to their internal phase transitions then there could be errors in the calculated cluster decay rates owing to simulation trajectories being trapped within a portion of phase space corresponding to some internal solid structure. ${ }^{70,71}$ Upon closer inspection however, the 38 -atom argon cluster in the study by Calvo et al ${ }^{70}$ possess less than $10 \%$ of nonliquid structure at the highest temperature considered of $\sim 36 \mathrm{~K}$. Furthermore, their reported phase change occurs at a temperature of $\sim 22 \mathrm{~K}$, well below the range of temperatures employed in the current study. With respect to the study of the 28 -atom argon cluster by Curotto, ${ }^{71}$ their results suggest a complete absence of non-liquid structure above a temperature of $\sim 24 \mathrm{~K}$.

To conclude, this study finds that despite the slight expansion seen in the argon clusters at higher simulation temperatures, there is little effect upon the extent of the cluster boundary. With regard to the effect of the carrier gas upon cluster stability, no great changes in cluster decay rates or lifetimes are detected and thus provides justification for existing calculations using isolated clusters as approximations for real systems. However, the results do suggest that there may be a slight stabilising effect due to the presence of the carrier gas, most notably for the lower of the two helium gas densities and pressures employed here. The fact that a smaller stabilising effect is seen for the higher density and pressure carrier gas may lend support to the finding by Ford ${ }^{48}$ of the existence of a non-linear pressure dependence yet to be verified by experiments. Ultimately, many more calculations than have been performed in this study will be needed in order to build up a more complete picture of the carrier gas pressure and density dependence of cluster stabilities. 
1 D. H. E. Gross, Microcanonical Thermodynamics (World Scientific, Singapore, 2001).

2 K. Huang, Statistical Mechanics (John Wiley and Sons, 1987), 2nd ed.

3 J. Reiss, Methods of Thermodynamics (Dover Publications, New York, 1996).

4 D. Kashchiev, Nucleation: Basic Theory with Applications (Butterworth-Heinemann, 2000).

5 J. Frenkel, Kinetic Theory of Liquids (Oxford University Press, 1946).

${ }^{6}$ M. Bakhtar, M. Ebrahimi, and R. A. Webb, Proc. Instn Mech. Engrs. 209(C2), 115 (1995).

7 M. Bakhtar, M. Ebrahimi, and B. O. Bamkole, Proc. Instn Mech. Engrs. 209(C3), 169 (1995).

8 F. Bakhtar and M. R. Mahpeykar, Proc. Instn Mech. Engrs., Part C: J. Mechanical Engineering Science 209(C3), 195 (1997).

9 M. Blander and J. Katz, Amer. Inst. Chemical Engineers 21, 833 (1975).

10 M. Volmer and A. Weber, Z. Physikalische Chemie 119, 277 (1926).

11 R. Becker and W. Döring, Ann. Physik. (Leipzig) 24, 719 (1935).

12 J. Zeldovich, Soviet Physics JETP 12, 525 (1942).

13 F. B. Sprow and J. M. Prausnitz, Trans. Faraday Soc. 62, 1097 (1966).

14 I. J. Ford, Phys. Rev. E 56, 5615 (1997).

15 P. E. Wagner and R. Strey, Journal of Physical Chemistry 85, 2694 (1981).

16 R. C. Miller, R. J. Anderson, J. L. Kassner, and D. E. Hagen, J. Chem. Phys. 78, 3204 (1983).

17 Y. Viisanen, R. Strey, and H. Reiss, J. Chem. Phys. 99, 4680 (1993).

18 Y. Viisanen, R.Strey, and H. Reiss, J. Chem. Phys. 112, 8205 (2000).

19 I. J. Ford, Proc. Instn. Mech. Engrs. 218, 883 (2004).

20 A. Fladerer and R. Strey, J. Chem. Phys. 124, 164710 (2006).

21 W. G. Courtney, J. Chem. Phys. 35, 2249 (1961).

${ }^{22}$ L. Dufour and R. Defay, Thermodynamics of Clouds (Academic, New York, 1963).

23 M. Blander and J. L. Katz, J. Stat. Phys. 4, 55 (1972).

24 J. Lothe and G. M. Pound, J. Chem. Phys. 36, 2080 (1962).

${ }^{25}$ H. Reiss, W. K. Kegel, and J. L. Katz, J. Chem. Phys. 102, 8548 (1998).

26 P. Schaaf, B. Senger, J.-C. Voegel, R. K. Bowles, and H. Reiss, J. Chem. Phys. 114, 8091 (2001).

27 S. A. Harris and I. J. Ford, J. Chem. Phys. 118, 9216 (2003).

28 I. J. Ford and S. A. Harris, J. Chem. Phys. 120, 4428 (2004).

29 D. W. Oxtoby, J. Phys.: Condens. Matter 4, 7627 (1992).

30 A. Laaksonen, V. Talanquer, and D. W. Oxtoby, Annu. Rev. Phys. Chem. 46, 489 (1995).

31 K. Yasuoka and M. Matsumoto, J. Chem. Phys. 109, 8451 (1998).

32 P. R. ten Wolde, M. J. Ruiz-Montero, and D. Frenkel, J. Chem. Phys. 110, 1591 (1999).

33 X.-M. Bai and M. Li, J. Chem. Phys. 122, 224510 (2005).

34 R. Marek and J. Straub, Int. J. Heat Mass Transfer 44, 39 (2001). 
T. Kraska, J. Chem. Phys. 124, 054507 (2006).

36

J. L. Katz, C.-H. Hung, and M. Krasnopoler, Atmospheric Aerosols and Nucleation (Springer, Berlin, 1988), p. 356.

37 J. L. Katz and J. A. F. abd V. Chakarov, Nucleation and Atmospheric Aerosols (Deepak, Hampton, 1992), p. 11.

38 R. H. Heist, M. Janjua, and J. Ahmed, J. Chem. Phys. 98, 4443 (1994).

39 R. H. Heist, J. Ahmed, and M. Janjua, J. Chem. Phys. 99, 375 (1995).

40 O. V. Vasil'ev and H. Reiss, J. Chem. Phys. 105, 2946 (1996).

41 O. V. Vasil'ev and H. Reiss, Phys. Rev. E 54, 3950 (1996).

42 V. M. Novikov, O. V. Vasil'ev, and H. Reiss, Phys. Rev. E 55, 5743 (1997).

43 A. Bertelsmann, R. Stuczyski, and R. H. Heist, J. Chem. Phys. 100, 9762 (1996).

44 D. Kane and M. S. El-Shall, J. Chem. Phys. 105, 7617 (1997).

45 A.-P. Hyvärinen, D. Brus, V. Ždímal, J. Smolík, M. Kumala, Y. Viisanen, and H. Lihavainen, J. Chem. Phys. 124, 224304 (2006).

46 G. Wilemski, B. E. Wyslouzil, M. Gauthier, and M. B. Frish, Nucleation and Atmospheric Aerosols (Deepak, Hampton, 1992), p. 23.

47 P. E. Wagner, R. Strey, and Y. Viisanen, Nucleation and Atmospheric Aerosols (Deepak, Hampton, 1992), p. 27.

48 I. J. Ford, J. Aerosol Sci. 23, 447 (1992).

49 D. W. Oxtoby and A. Laaksonen, J. Chem. Phys. 102, 6846 (1995).

50 G. Narsimhan and E. Ruckenstein, J. Colloid Interface Sci. 128, 549 (1989).

51 E. Ruckenstein and B. Nowakowski, J. Colloid Interface Sci. 137, 583 (1990).

52 B. Nowakowski and E. Ruckenstein, J. Chem. Phys. 94, 1397 (1991).

53 B. Nowakowski and E. Ruckenstein, J. Chem. Phys. 94, 8487 (1991).

54 C. W. Gardiner, Handbook of Stochastic Methods (Springer-Verlag, Berlin Heidelberg, 1985).

55 H. Reiss, J. L. Katz, and E. R. Cohen, J. Chem. Phys. 48, 5553 (1968).

56 S. M. Kathmann, G. K. Schenter, and B. C. Garrett, J. Chem. Phys. 111, 4688 (1990).

57 F. H. Stillinger, J. Chem. Phys. 38, 1486 (1963).

58 H. Reiss, A. Tabazadeh, and J. Talbot, J. Chem. Phys. 92, 1266 (1990).

59 DL-POLY molecular simulation program, written by W. Smith and T. R. Forester, copyright The Council for the Central Laboratory of the Research Councils, Daresbury Laboratory, nr. Warrington (1996).

60 J. O. Hirschfelder, C. F. Curtiss, and R. B. Bird, Molecular Theory of Gases and Liquids (Wiley, 1964).

61 A. Michels, H. Wijker, and H. Wijker, Physica 15, 627 (1949).

62 A. Michels and H. Wouters, Physica 8, 923 (1941).

63 W. M. Haynes, Cryogenics 18, 621 (1978).

64 R. B. Stewart and T. Jacobsen, J. Phys. Chem. Ref. Data 18, 639 (1989).

65 F. Mandl, Statistical Physics (Wiley, 1988), 2nd ed. 
66 K. Clusius and A. Frank, Z. Elektrochem. 49, 308 (1943).

67 C. Gladun, Cryogenics 11, 205 (1971).

68 G. N. Brown and W. T. Ziegler, Adv. Cryog. Eng. 25, 662 (1980).

69 I. Kusaka, Phys. Rev. E 73, 031607 (2006).

70 F. Calvo, J. P. Neirotti, D. L. Freeman, and J. D. Doll, J. Chem. Phys. 112, 10350 (2000).

71 E. Curotto, J. Chem. Phys. 114, 10702 (2001). 
Table I: Parameters for the Lennard-Jones potential ${ }^{60}$ of $\operatorname{argon}^{61}$ and helium, ${ }^{62}$ with the argon-helium interaction parameters calculated using the Lorentz-Berthelot mixing rules $\epsilon_{A r H e}=\left(\epsilon_{A r A r} \epsilon_{H e H e}\right)^{1 / 2}$ and $\sigma_{A r H e}=\left(\sigma_{A r A r}+\sigma_{H e H e}\right) / 2$.

\begin{tabular}{|c|c|c|c|}
\hline$j$ & $k$ & $\epsilon_{j k} / \mathrm{kJmol}^{-1}$ & $\sigma_{j k} / \AA$ \\
\hline \hline $\mathrm{Ar}$ & $\mathrm{Ar}$ & 0.995581 & 3.4050 \\
\hline $\mathrm{He}$ & $\mathrm{He}$ & 0.084311 & 2.6000 \\
\hline $\mathrm{Ar}$ & $\mathrm{He}$ & 0.289721 & 3.0000 \\
\hline
\end{tabular}


Figure 1: A typical snapshot of the 50-atom argon cluster, here within a helium carrier gas at $0.67 \mathrm{kgm}^{-3}$.

Figure 2: Typical temperature evolution for the Argon cluster at $38 \mathrm{~K}$ throughout an NVE simulation, with average values (black line) calculated every 100 snapshots.

Figure 3: Potentials of mean force from all simulations for the (a) isolated and thermostatted argon clusters at (b) $0.57 \mathrm{kgm}^{-3}$ and (c) $6.70 \mathrm{kgm}^{-3}$ carrier gas densities.

Figure 4: Comparisons of the (a) radial distribution functions and (b) density profiles (centred about the centre of mass of each cluster) for the isolated cluster at different temperatures.

Figure 5: Radial force distributions from the three sets of MD simulations for the (a) isolated and thermostatted argon clusters at (b) $0.57 \mathrm{kgm}^{-3}$ and (c) $6.70 \mathrm{kgm}^{-3}$ carrier gas densities.

Figure 6: Potentials of the mean force from the three sets of MD simulations for the (a) isolated and thermostatted argon clusters at (b) $0.57 \mathrm{kgm}^{-3}$ and (c) $6.70 \mathrm{kgm}^{-3}$ carrier gas densities.

Figure 7: Radial distribution functions (centred about the centre of mass of each cluster) from the three sets of MD simulations for the (a) isolated and thermostatted argon clusters at (b) 0.57 $\mathrm{kgm}^{-3}$ and (c) $6.70 \mathrm{kgm}^{-3}$ carrier gas densities.

Figure 8: Argon cluster evaporation rates calculated using the kinetic theory of cluster decay, compared with that obtained using classical nucleation theory, and a recent MD study by Kraska. ${ }^{35}$ 


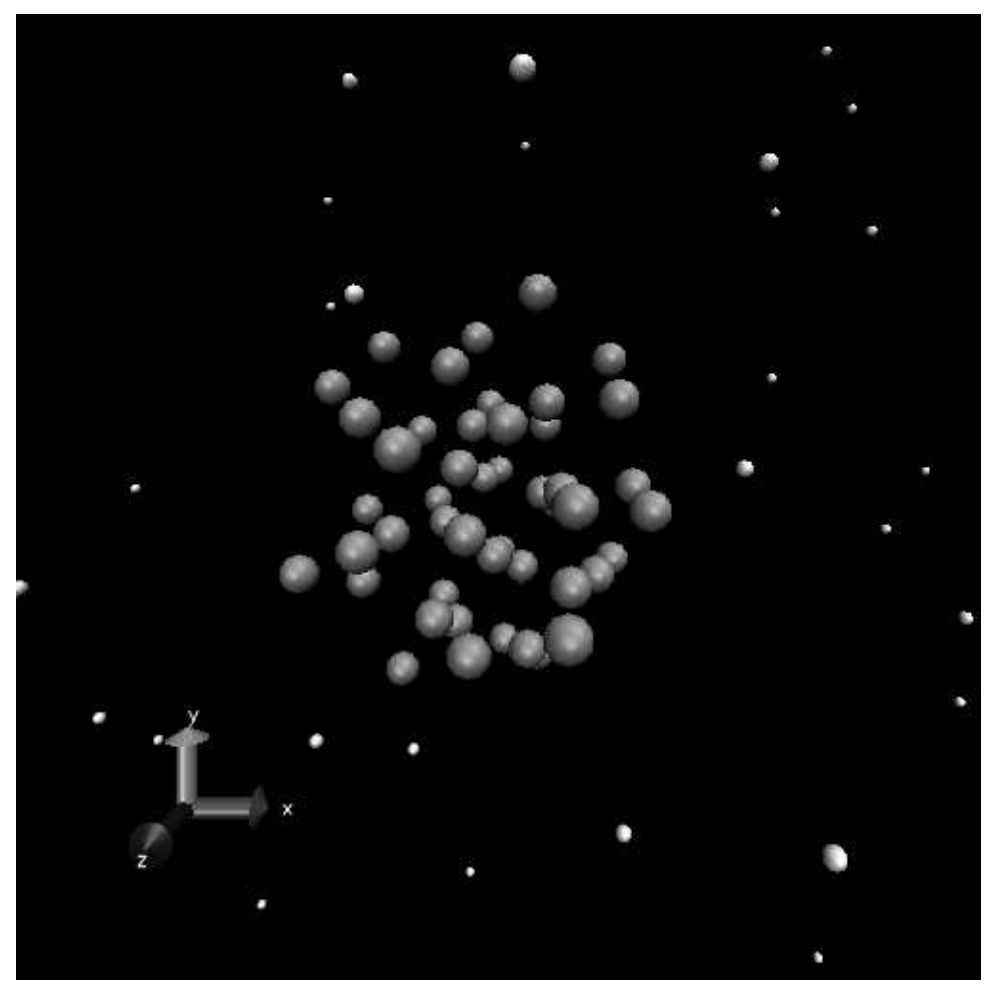

Figure 1: 


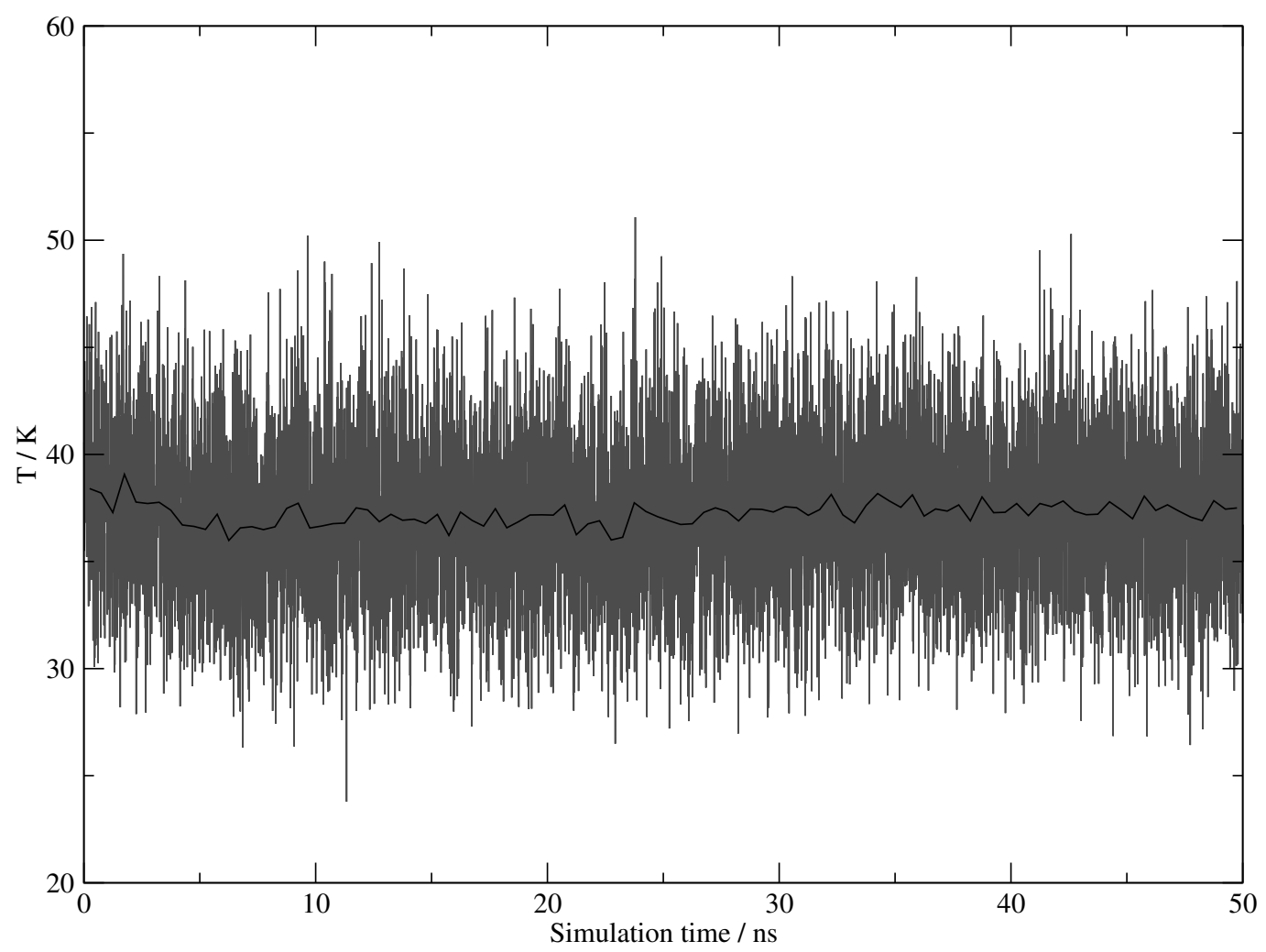

Figure 2: 
(a)

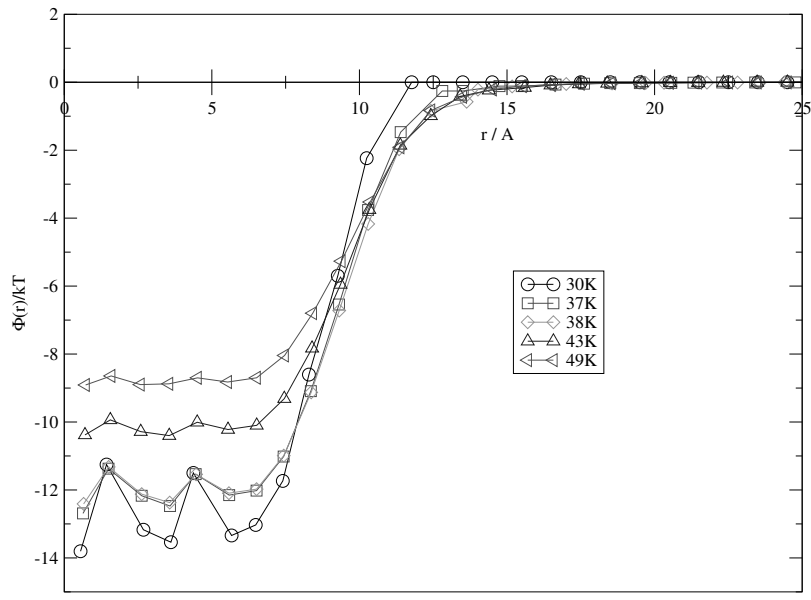

(b)

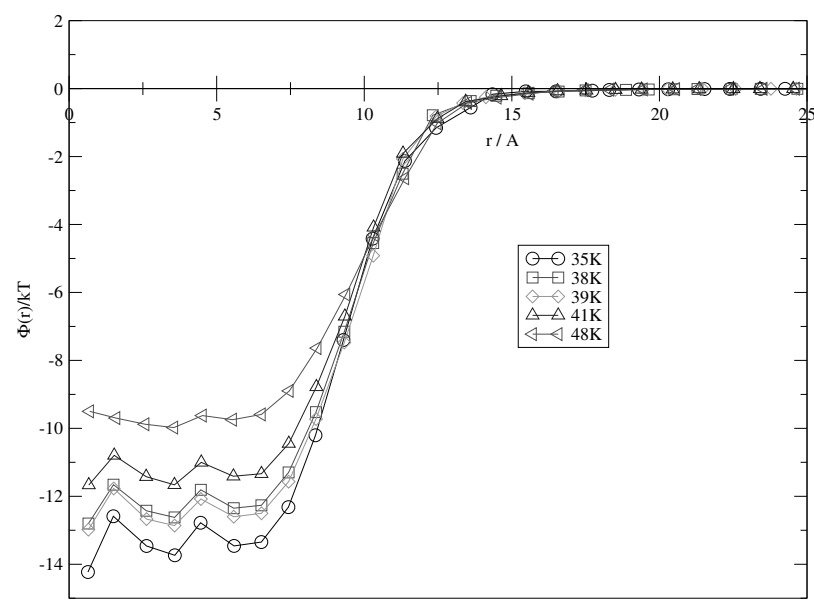

(c)

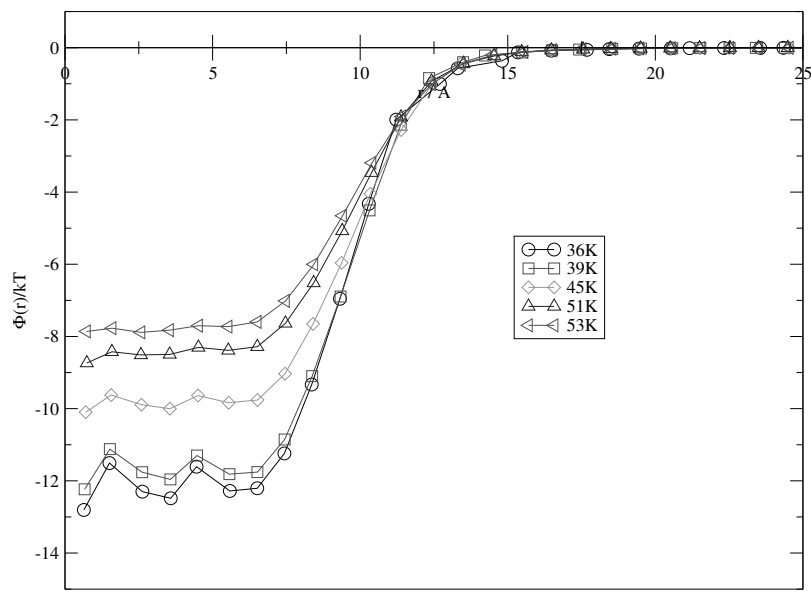

Figure 3: 
(a)

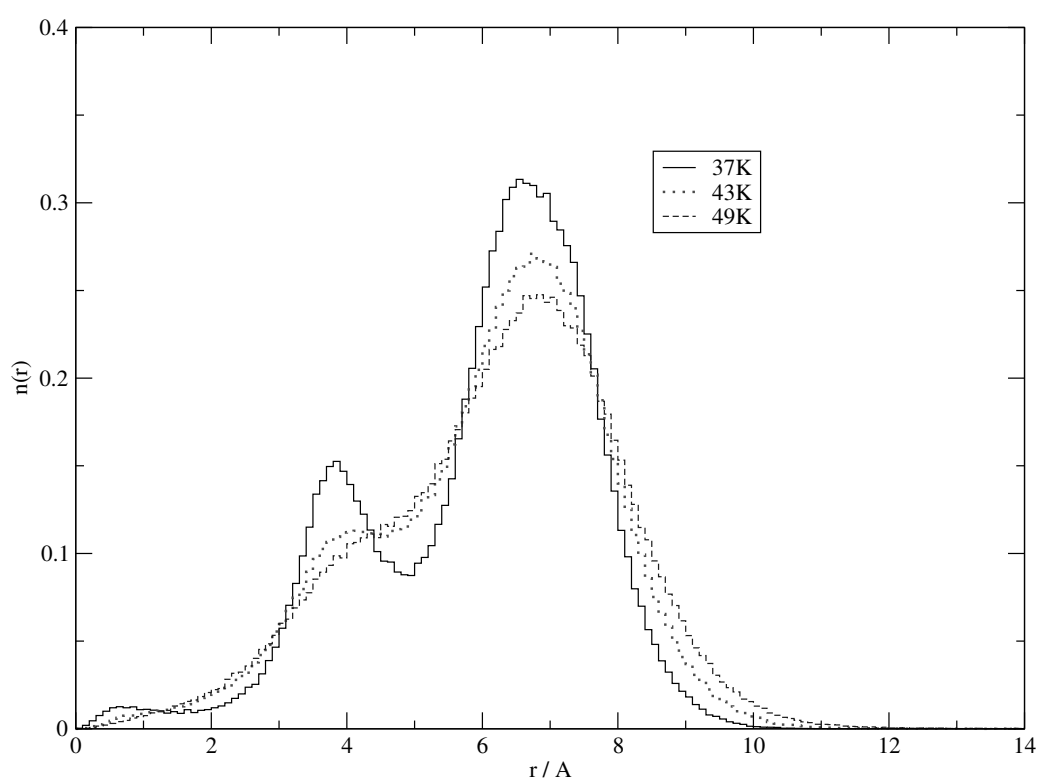

(b)

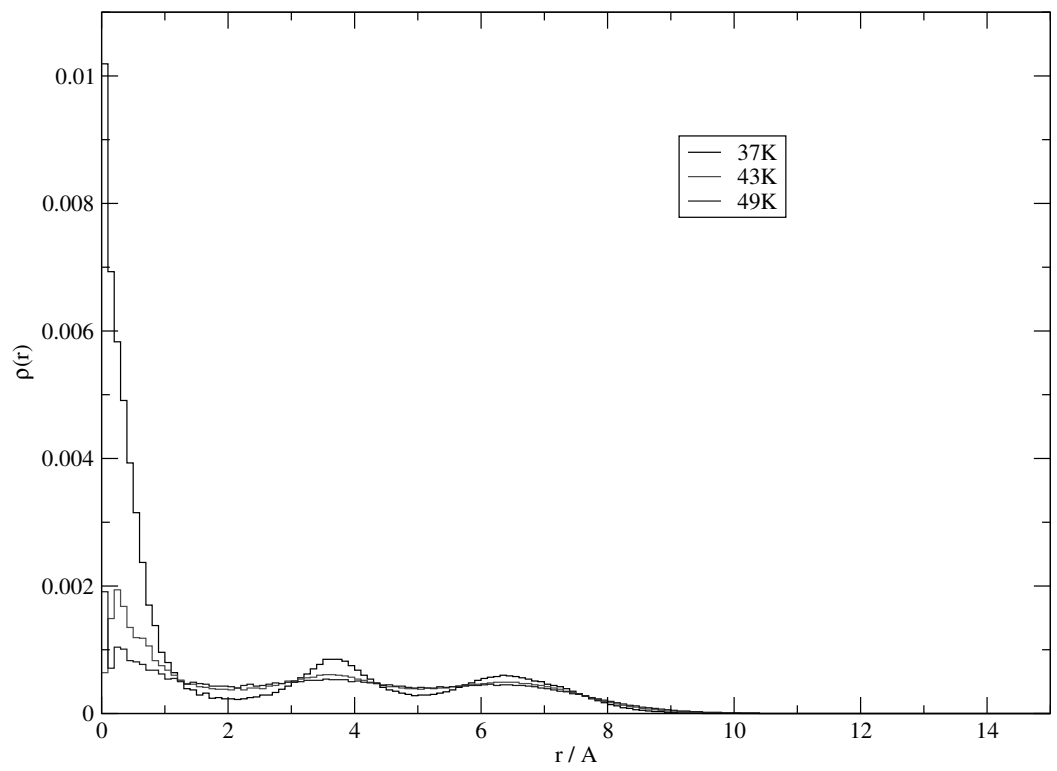

Figure 4: 
(a)

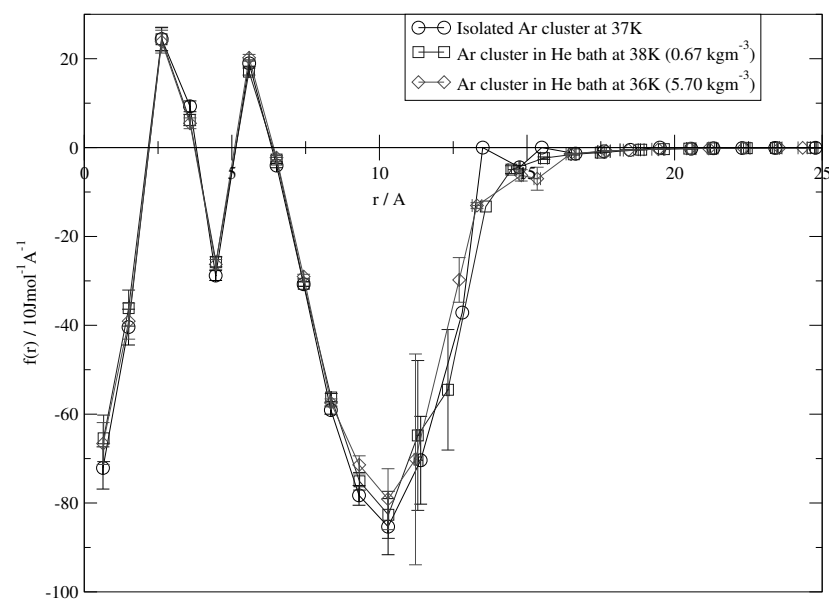

(b)

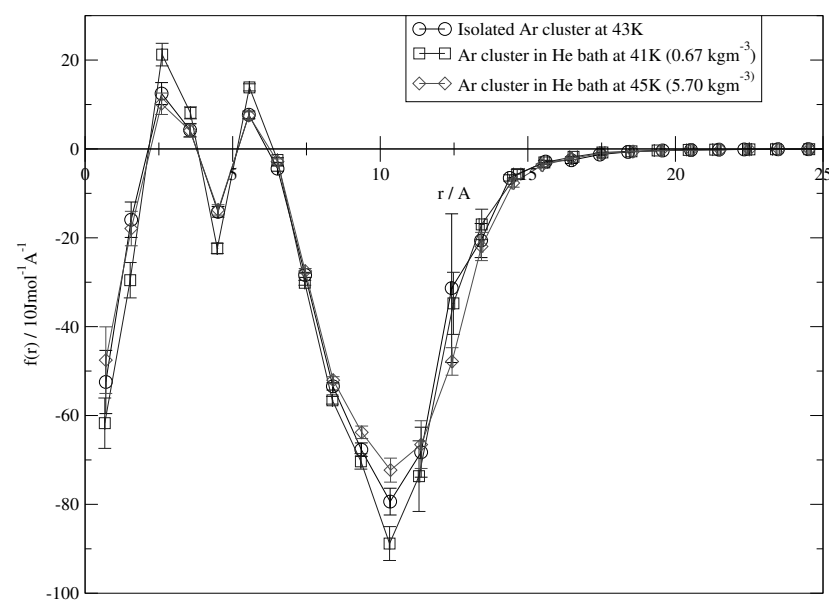

(c)

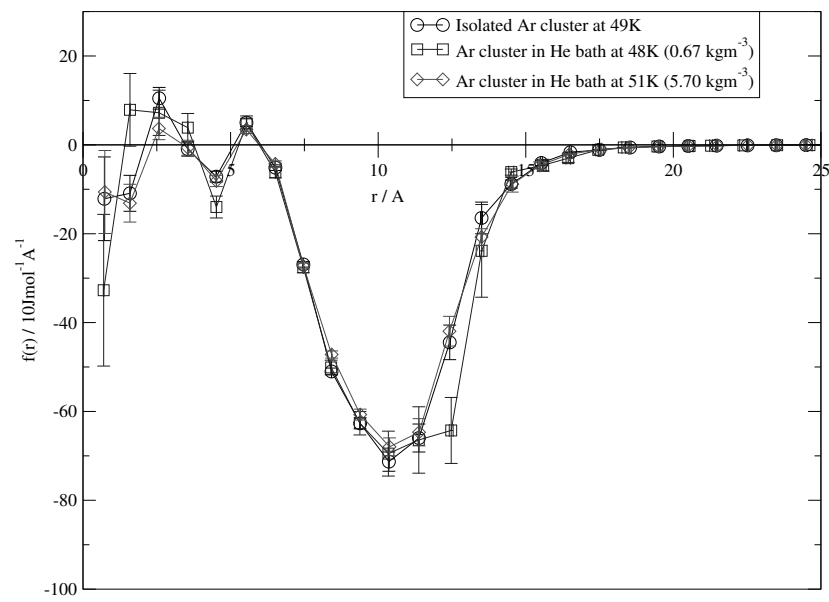

Figure 5: 
(a)

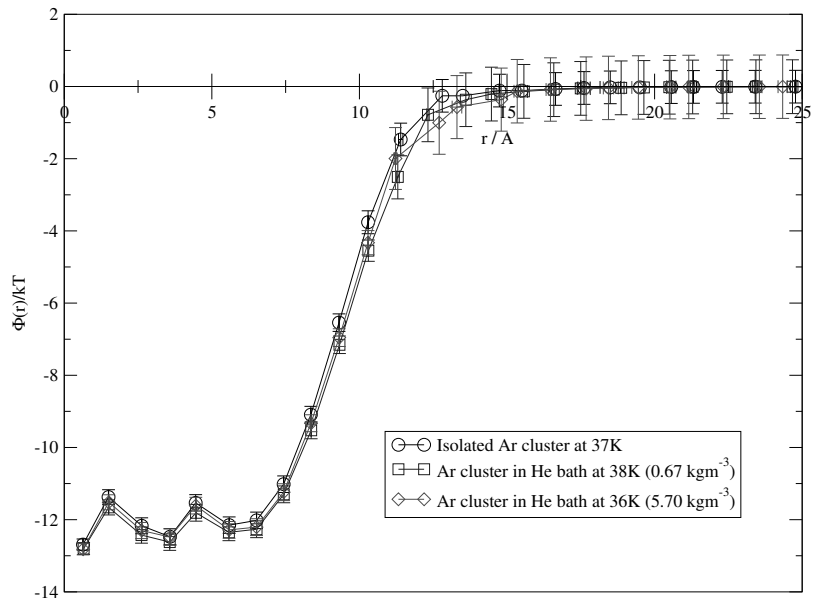

(b)

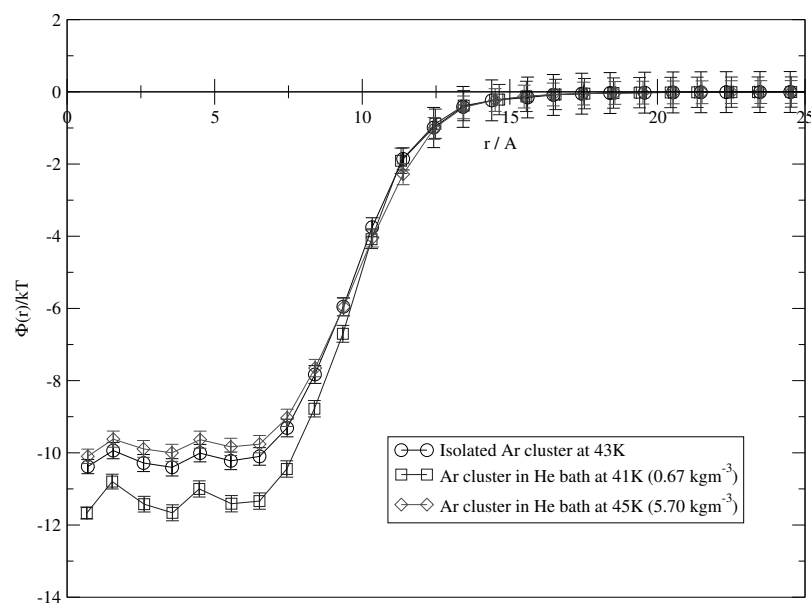

(c)

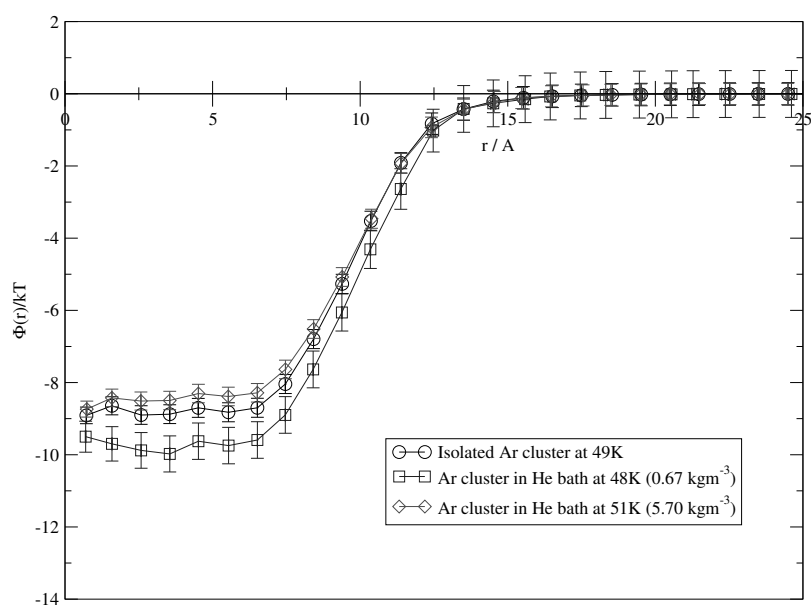

Figure 6: 
(a)

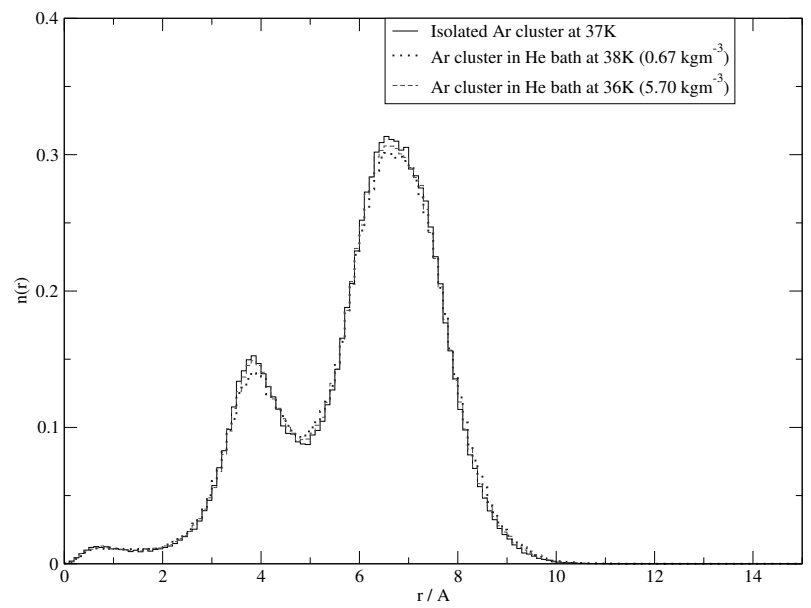

(b)

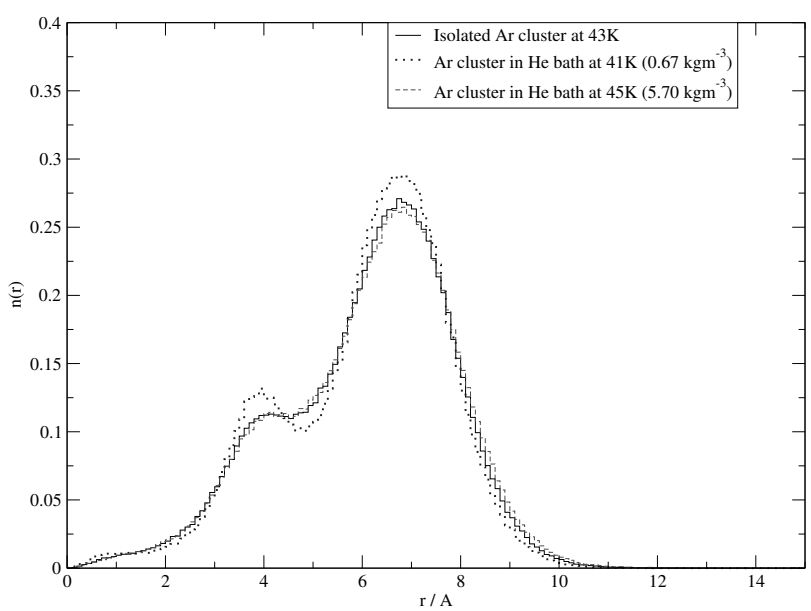

(c)

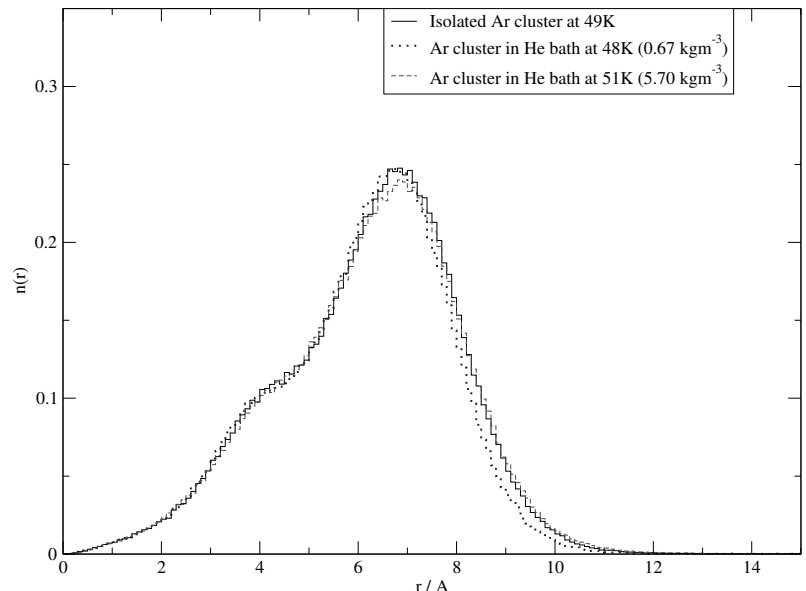

Figure 7: 


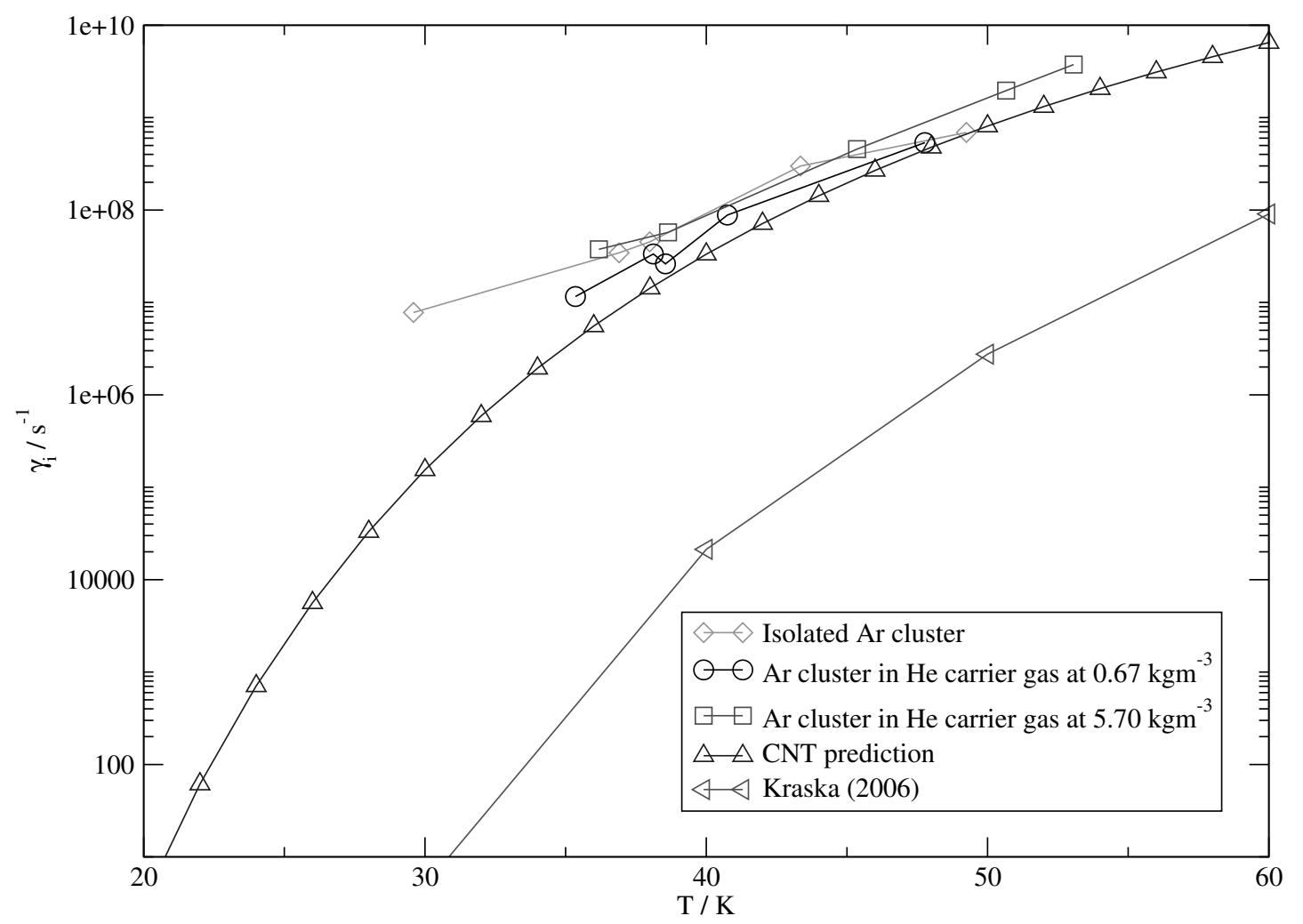

Figure 8: 